\title{
Experimental results on pulse magnetic field coupling into PCB and its suppression with shielding box
}

\author{
Longlong Chen ${ }^{1}$, Tianle $\mathrm{Li}^{2 *}$ and Chongqing Jiao ${ }^{2}$ \\ ${ }^{1}$ State Key Laboratory of Advanced Power Transmission Technology, Global Energy Interconnection Research Institute co., Ltd, \\ Beijing, 102209, China \\ ${ }^{2}$ State Key Laboratory of Alternate Electric Power System with Renewable Energy Sources, North China Electric Power University, \\ Beijing, 102206, China
}

\begin{abstract}
Due to the periodic switching operation of IGBT, there is a complex and high-intensity electromagnetic environment around the flexible DC converter valve. During this process, strong transit magnetic field caused by the turning on and off of large current can produce induced voltage on the nearby PCBs and even may affect the normal function of the PCBs. In this paper, the induced voltage in the output port on a PCB caused by a pulsed magnetic field is measured in laboratory. The effectiveness of a shielding box on suppressing the induced voltage and the magnetic field is also investigated experimentally. The results show that the induced voltage is about $12 \mathrm{mV}$ (in peak to peak value) for a $1.5 \mathrm{kA} / \mathrm{m}, 6.4 \mu$ s pulse magnetic field, and the shielding box has about $50 \mathrm{~dB}$ time-domain shielding effectiveness.
\end{abstract}

\section{Introduction}

The turn-on and turn-off process of a large number of IGBTs in the flexible DC converter valve needs to be controlled by a control circuit board. The board port is generally directly connected to the IGBT trigger pole or through a short cable[1]. The board is exposed to the electromagnetic field in the inner space of the converter valve, which can easily cause complicated distribution[23]. The shielding box has a good ability on the suppression of space electromagnetic disturbance. It can effectively shield the space electric field[4], but the shielding of the low-frequency magnetic field needs further research[5-7]. Inside the converter valve, the current peak is high, the electromagnetic coupling path is mixed, and the circuit board is close to the current path. Thus, the electromagnetic protection ability of the shielding box to the circuit board needs to be further evaluated.

The circuit board is generally composed of a power board, a central control board, and a driver board, all of which are printed circuit boards (PCB). Due to the loop and various components of the board, it can induce coupling voltage in an incident magnetic field. that is, space electromagnetic disturbance[8]. Based on the pulse magnetic field immunity test, this paper builds a test platform for the electromagnetic disturbance in the PCB port. The induced disturbance voltage to the transient pulse magnetic field with and without shielding was captured. By directly comparing the peak valve of the disturbance voltage, the ability of the shielding box to resist spatial magnetic field disturbance was verified.

\section{The test platform for the electromagnetic disturbance in the PCB port}

The test platform is mainly composed of a pulsed magnetic field generator, a magnetic field coil, an oscilloscope, a measuring cable, the test circuit board and the others.

- The pulse magnetic field generator model is EMS61000-9B, which can generate $6.4 / 16 \mu \mathrm{s}$ standard pulse current waveform.

- The magnetic field coil is a single-turn rectangular coil with a size of $1 \mathrm{~m} \times 1 \mathrm{~m}$. With the generator, a high magnetic field which peak value can reach $3.5 \mathrm{kA} / \mathrm{m}$ can be generated, which meets the requirements of the IEC 610004-9:2016.

- The measuring cable model is RG316, which has a good shielding effect to the space magnetic field. The introduced error is small, which can effectively ensure the accuracy of the voltage measurement of the output port on the board.

The experiment method is as follows: the pulse magnetic field generator is used to excite the magnetic field coil, a test pulse magnetic field is generated in the center of the coil, the circuit board is placed in the center of the coil, the voltage on the port is transmitted to the oscilloscope through the measuring cable, and the oscilloscope waveform is recorded. The laboratory equipment used for the measurement is shown in Figure 1. 


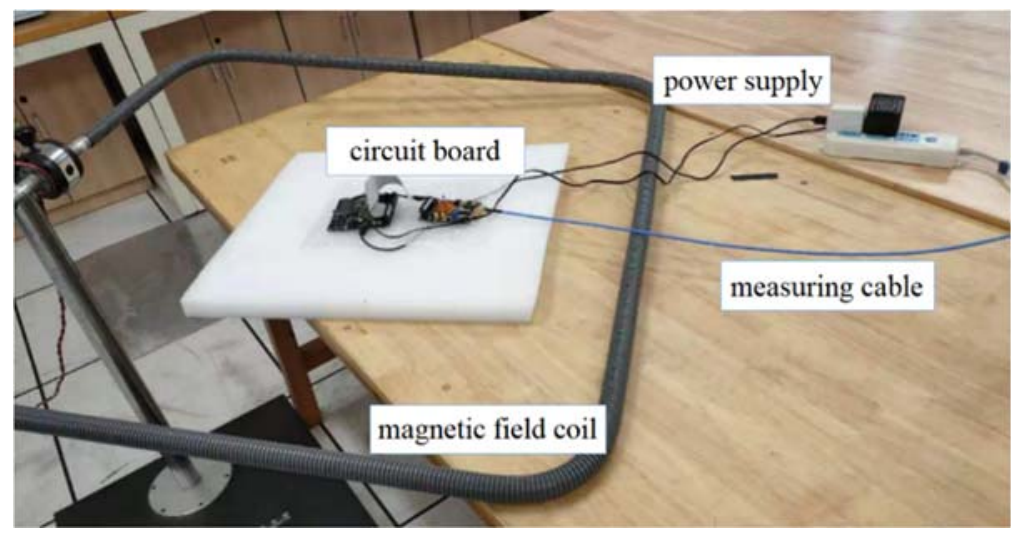

Figure 1. Laboratory setup

The applied pulse current excitation has a rise time of $6.4(1 \pm 30 \%) \mu \mathrm{s}$ and a duration time of $16(1 \pm 30 \%) \mu \mathrm{s}$, which has a wide frequency band. In the immunity test, when the peak value of the magnetic field pulse reaches $1 \mathrm{kA} / \mathrm{m}$, it can reach the level 5. However, in order to analysis the magnetic protection characteristics of the shielding box to the internal circuit board, the test magnetic field should be increased as much as possible on the condition of safety of the circuit board. Taking the above factors into consideration, the output voltage of the pulsed magnetic field generator was set to $3.5 \mathrm{kV}$. The current waveform in the magnetic field coil with a peak value of $1.6 \mathrm{kA}$ is shown in Figure 2.

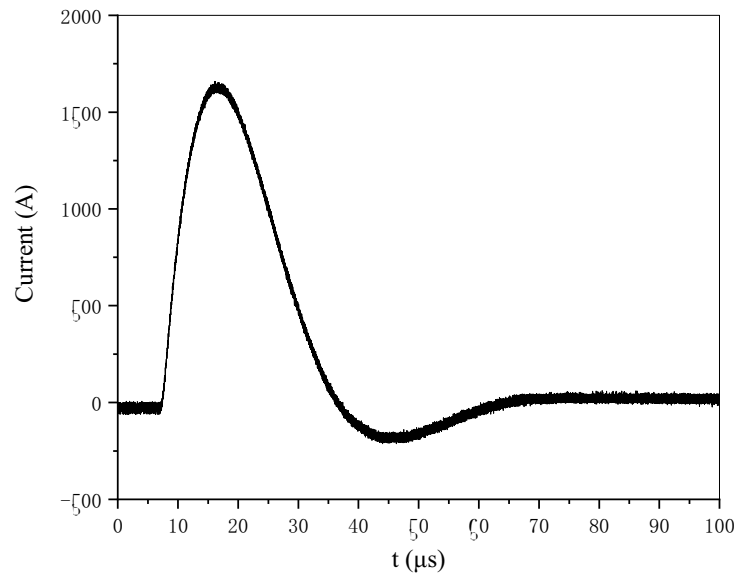

Figure 2. The current in the coil

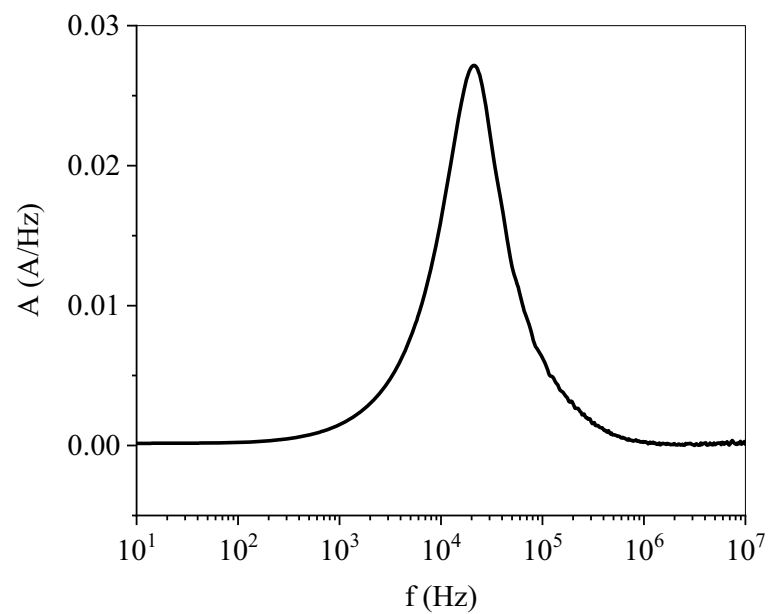

Figure 3. The spectral density of the current in the coil
In applications, the magnetic field at the center of the magnetic field coil is usually estimated by measuring the current flowing through the coil. The coil factor represents the ratio $(\mathrm{H} / \mathrm{I})$ between the test magnetic field and the current injected into the magnetic field coil. For the standard magnetic field coil used in this paper, the coil factor is 0.89 . According to the current waveform shown in figure 2 , the peak value of the magnetic field in the center of the magnetic field coil can reach $1.4 \mathrm{kA} / \mathrm{m}$. The spectral density of the test magnetic field is shown in Figure 3, it can be seen that the energy is mainly concentrated between $1 \mathrm{kHz}-500 \mathrm{kHz}$.

\section{Experimental results}

As mentioned above, the actual magnetic field in the converter valve is much higher than laboratory test magnetic field. But in the case of no magnetic saturation and no damage to the board, the disturbance voltage in the board port is proportional to the amplitude of the external magnetic field[9], so this laboratory platform can be used for simulation experiment.

The specification of the shielding box studied in this paper is $225 \mathrm{~mm} \times 95 \mathrm{~mm} \times 40 \mathrm{~mm}$ ( $1 \mathrm{~mm}$ thickness), and the material is aluminum (conductivity $\sigma=3.8 \times 10^{7} \mathrm{~S} / \mathrm{m}$, relative permeability $\mu_{\mathrm{r}}=1$ ). One side has three apertures of $25 \mathrm{~mm} \times 8 \mathrm{~mm}$, one side has two apertures of $21 \mathrm{~mm} \times 13$ $\mathrm{mm}$ and one aperture of $22 \mathrm{~mm} \times 17 \mathrm{~mm}$, and one side has one aperture of $19 \mathrm{~mm} \times 11 \mathrm{~mm}$. The schematic diagram is shown in Figure 4.

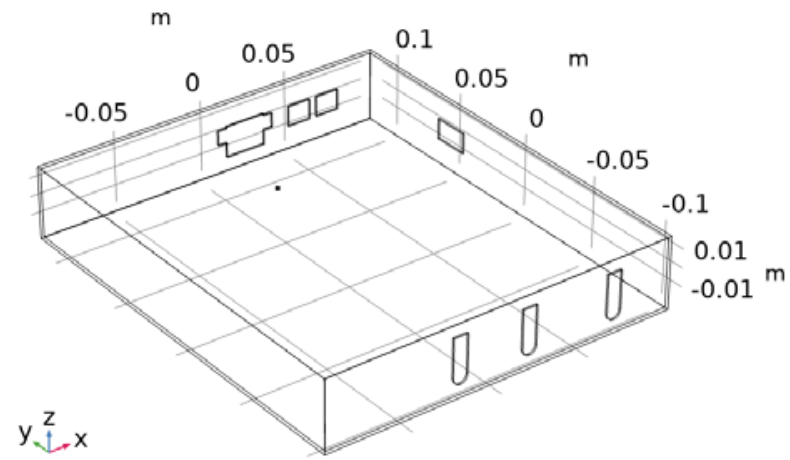

Figure 4. The specification of the shielding box 


\subsection{The induced voltage on $\mathrm{PCB}$ ports}

The board used is the AD9854 quadrature digital signal generator, which includes a high-speed DDS module and a control board, and is a very flexible device with a wide range of applications. Driven by a high-stability clock, the device will generate a stable sinusoidal signal, which can be programmed to control its frequency, phase and amplitude. This paper mainly uses it to simulate the IGBT drive control board. Although the function and design of the board is different from the IGBT control board, this paper focuses on analysing the electromagnetic protection characteristics of the shielding box to the board. The experimental results still have certain reference value.

The induced voltage in the output port without shielding is shown in figure 5 when the board is not working. It can be seen from the figures that the circuit board can induce the pulse voltage waveform with the pulse magnetic field, the peak-to-peak value can reach $12 \mathrm{mV}$, and the curve trend is more consistent with the test magnetic field, which does not bend to Faraday's law. A large number of inductance and capacitance components make the waveform distortion.

When the board is enclosed in the aluminum shielding box, the induced voltage is shown in figure 6 . It can be seen that the induced voltage is almost zero. According to the test results, conclusions can be drawn as follows:

- When the circuit board is exposed to a high spatial magnetic field, due to the design of the board, the output port will induce disturbance voltage through the spatial coupling.

- The shielding box can effectively shield the spatial magnetic field. When the circuit board is placed in the shielding box, the induced disturbance voltage is greatly reduced.

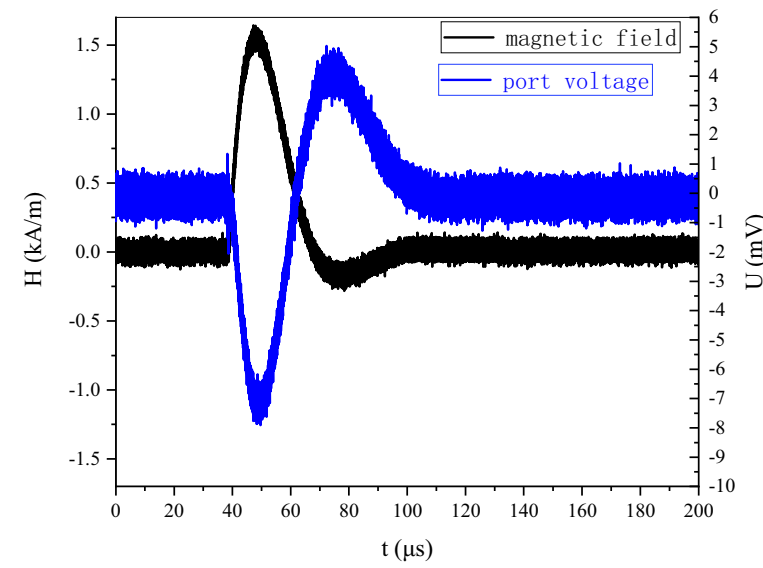

Figure 5. The induced voltage in the output port without shielding

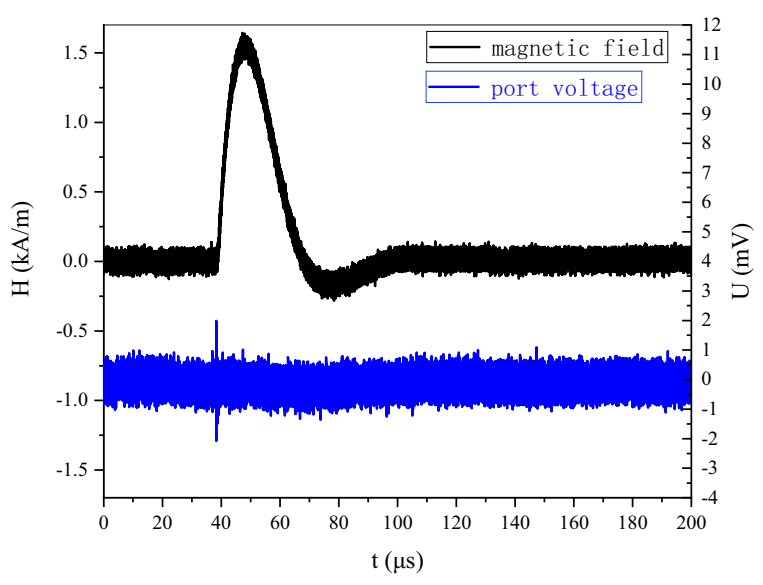

Figure 6. The induced voltage in the output port with shielding

Due to the difference of the disturbance magnetic field and the circuit board design, the laboratory experiment aims to provide reference data for the actual engineering. In the actual engineering, specific test should be done to get the induced disturbance voltage.

\subsection{The shielding effectiveness of the shielding box}

In the above chapter, the induced voltage in the port on the circuit board is low, which makes the analysis of the shielding effectiveness of the shielding box difficult. A near field probe is used to replace the circuit board, which is a single coil with $5 \mathrm{~cm}$ radius. The induced voltage in the probe without shielding is shown in figure 7 , with shielding is in figure 8 . It can be seen that the peak value is about $850 \mathrm{mV}$ without shielding, $2.5 \mathrm{mV}$ with shielding. The shielding effectiveness can be calculated by the ratio of the peak values, namely about $50 \mathrm{~dB}$. This value has met the engineering requirements.

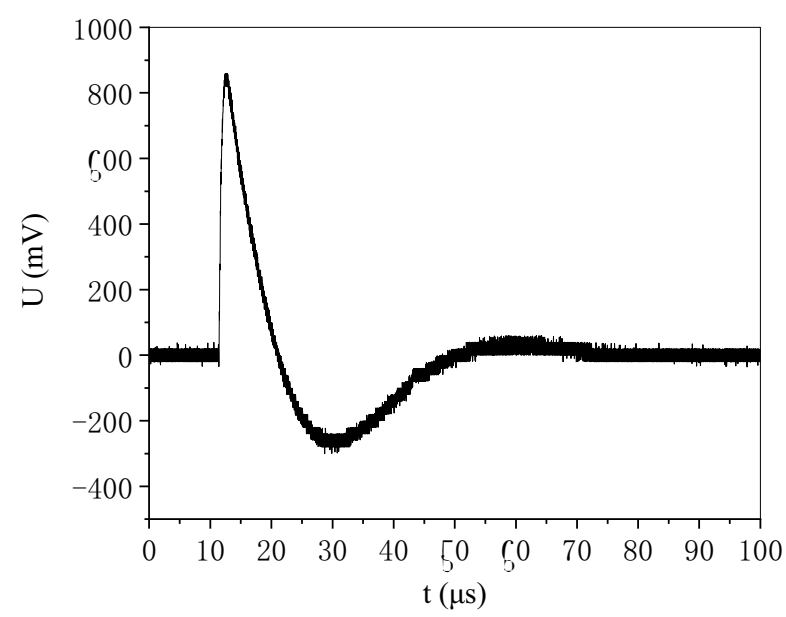

Figure 7. The induced voltage in the probe without shielding 


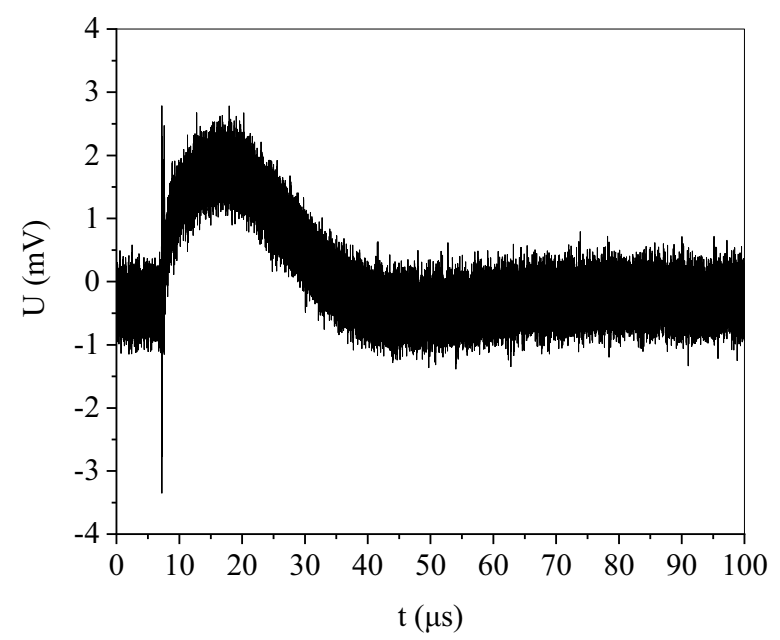

Figure 8. The induced voltage in the probe with shielding

\section{Conclusion}

By building test platform for the electromagnetic disturbance in the PCB port, the induced voltage in the output port on the circuit board is measured. There are some conclusions:

- The output port on the PCB will induce disturbance voltage through coupling a high transient magnetic field. The peak-to-peak value can reach $12 \mathrm{mV}$.

- The shielding box can reduce the disturbance voltage in the PCB port to a very small value.

- $\quad$ The shielding effectiveness of the shielding box can reach $50 \mathrm{~dB}$ against pulse magnetic field, which met the engineering requirements.

\section{Acknowledgments}

This project was supported by State Key Laboratory of Advanced Power Transmission Technology (GEIRISKL-2018-006).

\section{References}

1. Wang Yuhong, Shi Futong, Liu Chengzhuo, et al. (2020) Research on parameters of direct current circuit breaker for direct current grid. Science Technology and Engineering, 20(12): 4741-4747.

2. Wei Zhuo, Dong Chaoyang, $\mathrm{Hu}$ Siquan, et al. (2015) Valve control equipment of high voltage direct current transmission anti electronic magnetic interference analysis and design. Power Electronics, 49(12):8-12.

3. Liu Yi. (2012) The research of IGBT near-field electromagnetic disturbance characteristics. North China Electric Power University, Beijing, China.

4. Jiao Chongqi, Niu Shuai, Li Lin. (2015) Experiment study of power frequency electric and magnetic shielding effectiveness for composite materials. Transactions of China Electrotechnical Society, 30(10): 1-6.
5. Guillaume A., Johan P., Alain R., et al. (2012) Homogenization of composite panels from a nearfield magnetic shielding effectiveness measurement. IEEE Transactions on Electromagnetic Compatibility, 54(3): 700-703.

6. Hoeft LO., Hofstra JS. (1988) Experimental and theoretical analysis of the magnetic field attenuation of enclosures[J]. IEEE Transactions on Electromagnetic Compatibility, 30(3): 326-340.

7. Amin F., Mohamed B., Fabrice D., et al. (2015) A new methodology to predict the magnetic shielding effectiveness of enclosures at low frequency in the near Field. IEEE Transactions on Magnetics, 51(3): 800404.

8. Chen Jianhua. (2010) Study on signal integrity and EMC characteristics of PCB transmission lines. Xidian University, Xi'an, China.

9. FrederickM.T., MichelV.I., Torbjorn,K., (2009) EMC Analysis Methods and Computational Models. Beijing University of Posts and Telecommunications Press, Beijing, China. 